saturation was reached. We extracted information on geographical coverage and time periods. Quality assessment was carried out to rate and filter policies according to five criteria: 1. Potential for policy to affect maternal and child health outcomes; 2. Implementation variation across the UK; 3. Population reach and expected effect size; 4. Ability to identify exposed group in administrative data; 5. Potential to affect health inequalities. Finally, a consensus workshop was undertaken with experts to prioritise the included policies based upon existing knowledge.

Results The systematic search found 335 policies and 306 strategy documents. After filtering, 88 policies were found to vary across the 4 UK nations. Domains include: 32 welfare, 23 education, 20 health, 7 environment, 4 housing and 2 employment policies. Policies were mainly excluded due to criteria $2,3 \& 4$. The consensus workshop identified three policies as suitable candidates for quasi-experimental evaluation using administrative data: Pregnancy grants (welfare), Early Years Childcare (education) and Universal Credit (welfare). These policies are broadly similar across countries but differ in timing of implementation and details of target populations, offering opportunities for evaluation of effectiveness. For example, pregnancy grants are given to first born children in all UK countries, but only to second and subsequent children in Scotland.

Conclusion Through applying systematic review methods to a policy search, we identified some valuable opportunities to evaluate upstream impacts on mother and child outcomes. However, many potentially impactful policies did not meet the criteria for quasi-experimental evaluation, which could lead to the inverse evidence law. This could be ameliorated by better access to administrative data (e.g. on eligibility criteria), staged implementation of future policies (affording greater cross-country variation) or alternative evaluation methods (e.g. simulations).

\section{OP76 HOSPITAL-BASED PREVENTATIVE HEALTH SERVICES FOR PEOPLE EXPERIENCING HOMELESSNESS: SYSTEMATIC REVIEW AND NARRATIVE SYNTHESIS}

\footnotetext{
${ }^{1}$ Serena Luchenski*, ${ }^{1}$ Joanna Dawes, ${ }^{2}$ Robert Aldridge, ${ }^{3}$ Shema Tariq, ${ }^{1}$ Fiona Stevenson, ${ }^{1}$ Andrew Hayward. 'Institute of Epidemiology and Healthcare, University College London, London, UK; ${ }^{2}$ Institute of Health Informatics, University College London, London, UK; ${ }^{3}$ Institute of Global Health, University College London, London, UK
}

\subsection{6/jech-2021-SSMabstracts.76}

Background Preventative health services, such as screening, vaccinations, and referrals to health and social services, improve health outcomes and reduce healthcare utilisation, costs, and inequities. People experiencing homelessness have significant unmet needs, but data are lacking on preventative health service provision. We aimed to review literature on hospital-based preventative health services for people experiencing homelessness.

Methods We systematically searched MEDLINE, Embase, PsycINFO, HMIC, CINAHL, Web of Science, and The Cochrane Library. We hand-searched the bibliographies and citing references of included studies. We included experimental and observational quantitative studies involving preventative health services in emergency departments or inpatient hospital settings from 1999-2019. The population included adults experiencing homelessness in high income countries. We included outcomes for health, social factors, healthcare utilisation, and healthcare costs. We managed studies in Endnote and extracted data using a standardised spreadsheet. We assessed quality and bias using the 'Quality Assessment Tool for Quantitative Studies' and narratively synthesised findings.

Results We identified 7935 articles from searches and reviewed 149 full text articles. Thirty-two met our eligibility criteria and were conducted in the USA $(n=15)$, UK $(n=9)$, Canada $(n=4)$, and Australia $(n=4)$. Sixteen studies were undertaken in emergency departments, 13 in inpatient wards, and 3 were conducted in both settings. We identified eight intervention categories: 1) homelessness screening, 2) case management, 3) screening, treatment initiation and referrals, 4) vaccinations, 5) discharge planning, 6) assistance with social needs, 7) pharmacological treatment, and 8) psychosocial services. Most studies described multi-component interventions. Results showed improvements in housing status, mental health, quality of life, and uptake of vaccinations and screening. Some studies reported successful integration with follow-up services, while others reported poor rates of onward care. Studies tended to report reductions in unplanned healthcare utilisation and costs, though not consistently. None showed harms. The overall strength of the evidence was weak to moderate with few randomised controlled trials.

Discussion Hospital-based preventative health services can improve housing status and health and may reduce unplanned healthcare utilisation and costs for people experiencing homelessness. Definitive data are lacking for effective integration across healthcare systems. Policy-makers and practitioners should consider providing hospital-based preventative services to tackle unmet needs and health inequities. Our study is limited by the lack of qualitative, grey literature, and non-English studies. Future research should investigate barriers and levers for successful implementation of hospital-based preventative health services and the integration of hospitals with primary care and other services.

\section{OP77 SOCIOECONOMIC DISADVANTAGE AND ETHNICITY ARE ASSOCIATED WITH LARGE DIFFERENCES IN COGNITIVE ABILITIES THAT UNDERLIE CHILDREN'S EDUCATIONAL OUTCOMES: ANALYSIS OF A PROSPECTIVE BIRTH COHORT STUDY}

${ }^{1}$ Kate E Mooney*, ${ }^{1}$ Kate E Pickett, ${ }^{2,3}$ Katy Shire, ${ }^{4}$ Richard J Allen, ${ }^{3,4}$ Amanda H Waterman. ${ }^{1}$ Health Sciences, University of York, York, UK; ${ }^{2}$ Bradford Institute for Health Research, Bradford Teaching Hospitals Foundation Trust, Bradford, UK; ${ }^{3}$ Centre for Applied Education Research, Wolfson Centre for Applied Health Research, Bradford, UK; ${ }^{4}$ School of Psychology, University of Leeds, Leeds, UK

\subsection{6/jech-2021-SSMabstracts.77}

Background Working memory (WM) is the ability to store and process information over short time periods. WM is a strong predictor of educational attainment; which is important for health and wellbeing across the lifecourse. There is controversy about whether or not different aspects of WM are affected by socioeconomic position, and very little known about how ethnicity may shape these relationships. We studied these interrelationships in a longitudinal study of children in Bradford, a multi-ethnic city with high levels of deprivation.

Methods Born in Bradford ( $\mathrm{BiB})$ is a prospective birth cohort study following the lives of over 13,500 children and their 